\title{
The geometry of determinant line bundles in noncommutative geometry
}

\author{
Partha Sarathi Chakraborty and Varghese Mathai*
}

\begin{abstract}
This article is concerned with the study of the geometry of determinant line bundles associated to families of spectral triples parametrized by the moduli space of gauge equivalence classes of Hermitian connections on a Hermitian finite projective module. We illustrate our results with some examples that arise in noncommutative geometry.
\end{abstract}

Mathematics Subject Classification (2000). 58B34; 46L87, 58G26.

Keywords. Regularity, spectral triples, determinant line bundles, index theorem for families, connections, gauge transformations, dimension spectrum.

\section{Introduction}

In the mid 1990s, Connes and Moscovici [4] formulated and proved a far-reaching local index theorem for spectral triples and introduced the correct definition of dimension in the noncommutative setting, where it is no longer an integer, but rather a subset of $\mathbb{C}$ which is called the dimension spectrum. This article aims to understand the stability of the dimension spectrum for families of spectral triples, and its implications on the existence of geometric structures on the determinant line bundle, such as the Quillen metric and the determinant section.

That is, we are concerned with the study of the geometry of determinant line bundles associated to families of spectral triples $(\mathcal{A}, \mathscr{H}, D)$ parametrized by the moduli space of gauge equivalence classes of Hermitian connections on a Hermitian finite projective module. Recall that spectral triples $(\mathcal{A}, \mathscr{H}, D)$ were introduced by Connes [2], as the defining object for a noncommutative manifold, where $\mathcal{A}$ is a unital $C^{*}$ algebra acting on a separable Hilbert space $\mathscr{H}, D$ an unbounded self-adjoint operator on $\mathscr{H}$ with compact resolvent, and $[D, a]$ is a bounded operator on $\mathscr{H}$ for all $a \in \mathcal{A}$.

Given a spectral triple $(\mathcal{A}, \mathcal{H}, D)$, a finite projective module $E$ over $\mathcal{A}$, a Hermitian structure on $E$ and any Hermitian connection $\nabla$ on $E$, a basic stability result implies that $\left(\operatorname{End}_{\mathcal{A}}(E), E \otimes_{\mathcal{A}} \mathscr{H}, D_{E, \nabla}\right)$ is again a spectral triple. The space of all Hermitian connections on $E$ is an affine space $\boldsymbol{C}_{E}$, and the gauge group $\mathcal{E}$ is defined

\footnotetext{
*Both authors gratefully acknowledge support from the Australian Research Council.
} 
to be a Lie subgroup of the group $\operatorname{Aut}_{\mathcal{A}}(E)$ of invertible elements in $\operatorname{End}_{\mathcal{A}}(E)$, such that $\mathscr{G}$ acts smoothly and freely on $\mathcal{C}_{E}$. Analogous to the classical case we define the determinant line bundle $\mathscr{L}$ of the index bundle for this family of spectral triples. This is a line bundle on the moduli space $\mathcal{C}_{E} / \mathscr{E}$ of gauge equivalent classes of Hermitian connections on $E$.

In order to state the hypotheses required to define the Quillen metric [12] and the determinant section of $\mathscr{L}$, we need to utilise the notions of regularity and simple dimension spectrum introduced in [4]. More precisely, we require the spectral triple $(\mathcal{A}, \mathcal{H}, D)$ to be regular with simple dimension spectrum and zero is not in the dimension spectrum. These are precisely the same assumptions made by Connes and Chamseddine [3] in their work on inner fluctuations of spectral actions, except that we do not need the assumption that zero is not in the spectrum of $D$, cf. §4.3. In this context, Higson [9] also treats the case when zero is in the spectrum of $D$, but our approach differs from his. Another technical result proved here is the stability property for regular spectral triples $(\mathcal{A}, \mathscr{H}, D)$, which says that $\left(\operatorname{End}_{\mathcal{A}}(E), E \otimes_{\mathcal{A}}\right.$ $\left.\mathscr{H}, D_{E, \nabla}\right)$ is again a regular spectral triple with simple dimension spectrum for any Hermitian structure on $E$ and any Hermitian connection $\nabla$ on $E$. For the application of these constructions to a mathematical understanding of anomalies, that is, the nonpreservation of a symmetry of the classical action by the full quantum action in a gauge field theory; see [1], [6].

The last section contains an explicit calculation of the Quillen metric and determinant section of the determinant line bundle of the index bundle for the family of spectral triples on the noncommutative torus parametrized by the moduli space of flat Yang-Mills connections on a free module of rank one, which were studied by Connes-Rieffel [5]. These are expressed in terms of the theta and eta functions on the moduli space, which is a torus.

In [11], Perrot has studied a K-theoretic index theorem for families of spectral triples parametrized by the moduli space of gauge equivalent classes of Hermitian connections on $E$. He makes the restrictive assumption that the gauge group $\mathscr{E}$ is contained in the unitary group of $\mathcal{A}$, which is unnecessary in our context here, and he also does not construct the determinant line bundle of the index bundle and its geometry, which is the main study in this article.

\section{Preliminaries}

In this section, we recall the construction of the determinant line on the Banach manifold of all bounded Fredholm operators acting between separable Hilbert spaces. This motivates the constructions used later in the article.

Recall that a Fredholm operator $T: \mathscr{H}_{0} \rightarrow \mathscr{H}_{1}$ between two infinite dimensional Hilbert spaces $\mathscr{H}_{0}, \mathscr{H}_{1}$, is a bounded linear operator such that $\operatorname{dim}(\operatorname{ker}(T))<\infty$ and $\operatorname{dim}(\operatorname{coker}(T))<\infty$. This implies in particular that $\operatorname{Im}(T)$ is a closed subspace of $\mathscr{H}_{1}$. Let $\mathscr{F}=\mathscr{F}\left(\mathscr{H}_{0}, \mathscr{H}_{1}\right)$ denote the space of all Fredholm operators between 
two infinite dimensional Hilbert spaces $\mathscr{H}_{0}, \mathscr{H}_{1}$. It follows from Atkinson's theorem that $\mathscr{F}$ is an open subset of the Banach space $\mathscr{B}\left(\mathscr{H}_{0}, \mathscr{H}_{1}\right)$, of all bounded linear operators between the two Hilbert spaces, which establishes in particular that $\mathscr{F}$ is a Banach manifold modeled on the Banach space $\mathscr{B}\left(\mathscr{H}_{0}, \mathscr{H}_{1}\right)$. It has countably many connected components labeled by, index: $\pi_{0}(\mathcal{F}) \stackrel{\cong}{\rightrightarrows} \mathbb{Z}$, where for $T \in \mathscr{F}$,

$$
\operatorname{index}(T)=\operatorname{dim}(\operatorname{ker}(T))-\operatorname{dim}(\operatorname{coker}(T)) .
$$

We want to review the construction of a smooth line bundle DET $\rightarrow \mathcal{F}$, called the determinant line bundle, such that $\operatorname{DET}_{T}=\Lambda^{\max }(\operatorname{ker}(T))^{*} \otimes \Lambda^{\max }(\operatorname{coker}(T))$. The obvious definition does not work since $\operatorname{dim}(\operatorname{ker}(T))$ jumps as $T$ varies smoothly.

This problem was essentially fixed in [12]. For the convenience of the reader we elaborate on his solution. Let $\mathrm{Gr}_{\text {fin }}$ denote the space of all finite dimensional subspaces of $\mathscr{H}_{1}$. Consider the open cover $\left\{U_{F}\right\}$ of $\mathscr{F}$, where $F \in \mathrm{Gr}_{\text {fin }}$ and $U_{F}=$ $\left\{T \in \mathscr{F} \mid \operatorname{Im}(T)+F=\mathscr{H}_{1}\right\}$. For $T \in U_{F}$, consider the exact sequence of finite dimensional vector spaces

$$
0 \rightarrow \operatorname{ker}(T) \rightarrow T^{-1} F \stackrel{T}{\rightarrow} F \rightarrow \operatorname{coker}(T) \rightarrow 0 .
$$

Since index is constant on smooth families, and the rank of $F$ is fixed on $U_{F}$, therefore the rank of $T^{-1} F$ is constant on smooth families in $U_{F}$. So $E^{F} \rightarrow U_{F}$ defined by $\mathcal{E}_{T}^{F}=T^{-1} F$, is a smooth vector bundle. The virtual vector bundle $\operatorname{INDEX}^{F} \rightarrow U_{F}$ is defined to be the pair $\left(\mathcal{E}^{F}, F\right)$, where $F$ denotes the trivial vector bundle over $U_{F}$ with fibre $F$.

Using the inner products on $\mathscr{H}_{i}, i=0,1$, the sequence in equation (1) splits, $\operatorname{ker}(T) \oplus F \cong T^{-1} F \oplus \operatorname{coker}(T)$, therefore

$$
\Lambda^{\max }(\operatorname{ker}(T))^{*} \otimes \Lambda^{\max }(\operatorname{coker}(T)) \cong \Lambda^{\max }\left(T^{-1} F\right)^{*} \otimes \Lambda^{\max } F .
$$

The determinant line bundle, $\mathrm{DET}^{F} \rightarrow U_{F}$, is defined as the smooth line bundle, $\operatorname{DET}^{F}=\operatorname{det}\left(\left(\operatorname{INDEX}^{F}\right)^{*}\right)$, i.e., $\operatorname{DET}^{F}=\Lambda^{\max }\left(E^{F}\right)^{*} \otimes \Lambda^{\max } F$.

Suppose that $T \in U_{E} \cap U_{F}$. Then we have the exact sequences

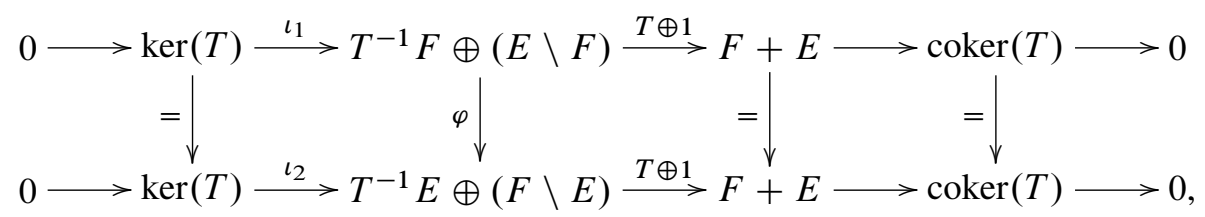

where $\varphi$ is uniquely defined so as to make the diagram commute. Here by $(E \backslash F)$ we mean the quotient $E /(E \cap F)$, etc. By the Five Lemma, the map $\varphi$ is an isomorphism, and therefore, $\mathrm{DET}^{F}=\Lambda^{\max }\left(\mathcal{E}^{F}\right)^{*} \otimes \Lambda^{\max }(E \backslash F)^{*} \otimes \Lambda^{\max }(F+E)$ and $\mathrm{DET}^{E}=$ $\Lambda^{\max }\left(E^{E}\right)^{*} \otimes \Lambda^{\max }(F \backslash E)^{*} \otimes \Lambda^{\max }(F+E)$ are naturally isomorphic via $\Lambda^{\max } \varphi \otimes 1$ over $U_{E} \cap U_{F}$. Here we have used the fact that $\Lambda^{\max }(V)^{*} \otimes \Lambda^{\max }(V)$ is canonically 
trivial for any vector bundle $V$. By the clutching construction, it follows that DET defines a smooth line bundle over the union $U_{E} \cup U_{F}$.

To prove that DET defines a smooth line bundle over the whole of $\mathcal{F}$, we need to show that on triple overlaps $U_{E} \cap U_{F} \cap U_{G}$, there are natural isomorphisms between the determinant line bundles $\mathrm{DET}^{E}, \mathrm{DET}^{F}$ and $\mathrm{DET}^{G}$. The proof is similar to the case of double overlaps. Suppose that $T \in U_{E} \cap U_{F} \cap U_{G}$. Then we have the exact sequences

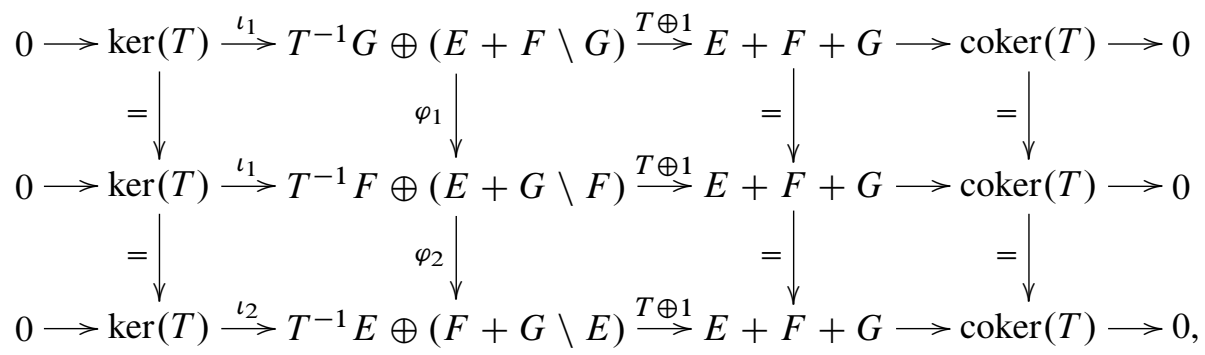

where $\varphi_{j}, j=1,2$, is uniquely defined so as to make the diagram commute. By the Five Lemma, the map $\varphi_{j}, j=1,2$, is an isomorphism, and therefore,

$$
\operatorname{DET}^{G}=\Lambda^{\max }\left(\mathcal{E}^{G}\right)^{*} \otimes \Lambda^{\max }(E+F \backslash G)^{*} \otimes \Lambda^{\max }(E+F+G)
$$

and

$$
\operatorname{DET}^{F}=\Lambda^{\max }\left(\mathcal{E}^{F}\right)^{*} \otimes \Lambda^{\max }(E+G \backslash F)^{*} \otimes \Lambda^{\max }(E+F+G)
$$

are naturally isomorphic via $\Lambda^{\max } \varphi_{1} \otimes 1$ over $U_{E} \cap U_{F} \cap U_{G}$, where we have used the fact that $\Lambda^{\max }(V)^{*} \otimes \Lambda^{\max }(V)$ is canonically trivial for any vector bundle $V$. Similarly,

$$
\operatorname{DET}^{F}=\Lambda^{\max }\left(E^{F}\right)^{*} \otimes \Lambda^{\max }(E+G \backslash F)^{*} \otimes \Lambda^{\max }(E+F+G)
$$

and

$$
\operatorname{DET}^{E}=\Lambda^{\max }\left(E^{E}\right)^{*} \otimes \Lambda^{\max }(F+G \backslash E)^{*} \otimes \Lambda^{\max }(E+F+G)
$$

are naturally isomorphic via $\Lambda^{\max } \varphi_{2} \otimes 1$ over $U_{E} \cap U_{F} \cap U_{G}$. Therefore DET ${ }^{G}$, $\mathrm{DET}^{F}$ and $\mathrm{DET}^{E}$ are canonically identified on the overlaps $U_{E} \cap U_{F} \cap U_{G}$. By the clutching construction, it follows that DET defines a consistent smooth line bundle over the union $U_{E} \cup U_{F} \cup U_{G}$. Since $E, F$ and $G$ are arbitrary finite dimensional subspaces of $\mathscr{H}_{1}$, it follows that DET defines a smooth line bundle on the Banach manifold $\mathscr{F}$.

It is a fact that $\mathcal{F}$ is homotopy equivalent to $\mathbb{Z} \times \mathrm{BU}(\infty)$, so that by Bott periodicity, $\pi_{2 j}(\mathcal{F}) \cong \mathbb{Z}$ and $\pi_{2 j+1}(\mathcal{F})=\{0\}$ for $j \geq 0$. So by Hurewicz's theorem, $H^{2}\left(\mathcal{F}_{0}, \mathbb{Z}\right) \cong \mathbb{Z}$, where $\mathcal{F}_{0}$ is the connected component of $\mathcal{F}_{F}$ consisting of Fredholm operators of index equal to zero. It is a fact that the first Chern class, $c_{1}(\mathrm{DET})$ is the generator of $H^{2}\left(\mathcal{F}_{0}, \mathbb{Z}\right) \cong \mathbb{Z}$. 


\section{Stability of spectral triples coupled to a Hermitian finite projective module with Hermitian connection}

The notion of a spectral triple was introduced by Connes [2] as the criteria defining a noncommutative spin geometry. A slightly weakened version, dropping the postulate of the existence of a real structure, was given by Moscovici [10] which was suited to describe more general Poincaré dual pairs of algebras, and in particular noncommutative $\operatorname{spin}^{c}$ geometries.

Let $A$ be a separable, unital $C^{*}$-algebra acting on a separable Hilbert space $\mathscr{H}$. Let $D$ be an unbounded self-adjoint operator on $\mathscr{H}$. Let $\mathcal{A}$ be a smooth unital subalgebra of $A$. Then $(\mathcal{A}, \mathscr{H}, D)$ is said to be a spectral triple if $D$ has compact resolvent and $[D, a]$ is a bounded operator on $\mathscr{H}$ for all $a \in \mathcal{A}$, and $(\mathscr{A}, \mathcal{H}, D)$ is said to be even if there is a self-adjoint involution on $\mathscr{H}$ with respect to which the action of $\mathcal{A}$ is even and $D$ is an odd operator. Otherwise the spectral triple is said to be $o d d$. We begin by considering the even case.

Let $E$ be a finite projective (right-)module over $\mathcal{A}$. A Hermitian structure on $E$ is a sesquilinear map $(\cdot, \cdot): E \times E \rightarrow A$ satisfying the following conditions:

(1) $(\xi a, \eta b)=a^{*}(\xi, \eta) b$ for all $\xi, \eta \in E$ and $a, b \in A$;

(2) $(\xi, \xi) \geq 0$;

(3) $E$ is self-dual with respect to $(\cdot, \cdot)$.

Consider the special case of the free $\mathcal{A}$-module $E_{0}=\mathcal{A}^{q}$ which has a canonical Hermitian structure given by $(\xi, \eta)=\sum_{1}^{q} \xi_{j}^{*} \eta_{j}$ for all $\xi=\left(\xi_{1}, \ldots, \xi_{q}\right), \eta=$ $\left(\eta_{1}, \ldots, \eta_{q}\right) \in E_{0}$.

Let $E$ be a finite projective (right) module over $\mathcal{A}$. If we write $E$ as a direct summand $E=e \mathcal{A}^{q}$ of a free module $E_{0}$, where $e \in M_{q}(\mathcal{A})$ is a self-adjoint projection. Then $E$ has a Hermitian structure, which is obtained by restricting to $E$ the Hermitian structure on $E_{0}$ defined above. That is, every finite projective module $E$ over $\mathcal{A}$ has a Hermitian structure.

Consider the $\mathcal{A}$-bimodule of 1 -forms on $\mathcal{A}, \Omega_{D}^{1}(\mathcal{A}):=\left\{\sum a_{j}\left[D, b_{j}\right] \mid a_{j}, b_{j} \in\right.$ $\mathcal{A}\}$. A Hermitian connection on $E$ is a $\mathbb{C}$-linear map $\nabla: E \rightarrow E \otimes_{\mathcal{A}} \Omega_{D}^{1}(\mathcal{A})$ satisfying the following:

(1) $\nabla(\xi a)=\nabla(\xi) a+\xi \otimes d a$ for all $\xi \in E, a \in \mathcal{A}$ (Leibniz property).

(2) $(\xi, \nabla \eta)-(\nabla \xi, \eta)=d(\xi, \eta)$ for all $\xi, \eta \in E$, where $d a=[D, a]$ for all $a \in \mathcal{A}$ (Hermitian property).

If $\nabla(\xi)=\sum \xi_{j} \otimes \omega_{j}$, with $\xi_{j} \in E, \omega_{j} \in \Omega_{D}^{1}(\mathcal{A})$, then $(\nabla \xi, \eta)=\sum \omega_{j}^{*}\left(\xi_{j}, \eta\right)$.

An example of a Hermitian connection is the Grassmannian connection $\nabla_{0}$ on $E=e \mathcal{A}^{q}$ given by $\nabla_{0}(\xi)=e d \xi$, where $d \xi=\left(d \xi_{1}, \ldots, d \xi_{q}\right)$. Also, any two Hermitian connections differ by a self-adjoint element of $\operatorname{End}_{\mathcal{A}}(E) \otimes_{\mathcal{A}} \Omega_{D}^{1}(\mathcal{A})$. That is, the space of all Hermitian connections on $E$ is an affine space $\mathcal{C}_{E}$ with associated vector space $\operatorname{End}_{\mathcal{A}}(E) \otimes_{\mathcal{A}} \Omega_{D}^{1}(\mathcal{A})$. 
Given a Hermitian finite projective module $E$ over $\mathcal{A}$, we can form the Hilbert space $E \otimes_{\mathcal{A}} \mathscr{H}$ by completing the algebraic tensor product with respect to the inner product $\left\langle\xi_{1} \otimes \eta_{1}, \xi_{2} \otimes \eta_{2}\right\rangle=\left\langle\eta_{1},\left(\xi_{1}, \xi_{2}\right) \eta_{2}\right\rangle$ for all $\xi_{j} \in E, \eta_{j} \in \mathscr{H}$.

Given such a pair $(E, \nabla)$, one can define a twisted operator $D_{E, \nabla}$ on the Hilbert space $E \otimes_{\mathcal{A}} \mathscr{H}$ by setting $D_{E, \nabla}(\xi \otimes \eta)=\xi \otimes D(\eta)+\nabla(\xi) \eta$ for all $\xi \in E, \eta \in \mathscr{H}$. Here, if $\nabla(\xi)=\sum \xi_{j} \otimes \omega_{j}$, then $\nabla(\xi) \eta=\sum \xi_{j} \otimes \omega_{j}(\eta) \in E \otimes_{\mathcal{A}} \mathscr{H}$.

We have the following result.

Lemma 2.1. Let $T$ be an unbounded self-adjoint operator on a Hilbert space $\mathscr{H}$ and suppose that there is an operator $S \in \mathcal{I}$, where $\mathcal{I}$ denotes either the ideal of all compact operators on $\mathscr{H}$ or a Schatten ideal on $\mathscr{H}$, such that $(i I+T) S-I \in \mathcal{I}$. Then $(i I+T)^{-1} \in \mathcal{I}$. Moreover, $(i I+R+T) S-I \in \mathcal{I}$ for any bounded self-adjoint operator $R$, and therefore $(i I+R+T)^{-1} \in \mathcal{I}$.

Proof. To prove this, note that

$$
(i I+T)^{-1}=S-K(i I+T)^{-1},
$$

where $K=(i I+T) S-I \in \mathcal{I}$. Therefore the right-hand side is in the ideal $\mathcal{I}$, as claimed. Since $R S \in \mathcal{I}$ for any bounded self-adjoint operator $R$, it follows that $(i I+R+T) S-I \in \mathcal{I}$, and therefore $(i I+R+T)^{-1} \in \mathcal{I}$, as claimed.

Proposition 2.2 (Stability of spectral triples I). Let $(\mathcal{A}, \mathcal{H}, D)$ be a spectral triple, $E$ be a finite projective $\mathcal{A}$-module with Hermitian structure and Hermitian connection $\nabla$. Then $\left(\right.$ End $\left._{\mathcal{A}}(E), E \otimes_{\mathcal{A}} \mathscr{H}, D_{E, \nabla}\right)$ is also a spectral triple. Moreover, if $(\mathcal{A}, \mathcal{H}, D)$ is $p$-summable, then so is $\left(\operatorname{End}_{\mathcal{A}}(E), E \otimes_{\mathcal{A}} \mathscr{H}, D_{E, \nabla}\right)$.

Proof. First observe that $\left(M_{N}(\mathbb{C}) \otimes \mathscr{A}, \mathbb{C}^{N} \otimes \mathscr{H}, 1 \otimes D\right)$ is a spectral triple for all natural numbers $N$, where $\mathbb{C}^{N} \otimes \mathscr{H} \cong \mathcal{A}^{N} \otimes_{\mathcal{A}} \mathscr{H}$. So the result is true when $E$ is a free module and with the trivial connection. For simplicity of notation, denote by $D$ the operator $I \otimes D$. An arbitrary connection on the free module $E$ is of the form $D+\sum_{i} a_{i}\left[D, a_{i}^{\prime}\right]$, where the term $\sum_{i} a_{i}\left[D, a_{i}^{\prime}\right], a_{i}, a_{i}^{\prime} \in \mathcal{A}$, is a bounded operator which we denote by $R$. Such perturbations of $D$ will be referred as inner fluctuations. The condition that the connection is Hermitian implies that $R$ is selfadjoint. By Lemma 2.1, it follows that $(\mathcal{A}, \mathscr{H}, D+R)$ is again a spectral triple, which is just the statement that spectral triples are stable under inner fluctuations.

Next we observe that there exists a projection $e \in M_{N}(\mathcal{A})$ for some natural number $N$ such that $E=e \mathcal{A}^{N}$. We want to show that $\left(\operatorname{End}_{\mathcal{A}}(E), E \otimes_{\mathcal{A}} \mathscr{H}, D_{E, \nabla_{0}}\right)$ is a spectral triple, where $\nabla_{0}=e[D, e]$ is the Grassmann connection and we have used the simplified notation. Consider the compact operator $S=e(i I+D)^{-1} e$. Then

$$
e(i I+D) e S=e(i I+D) e(i I+D)^{-1} e=e[(i I+D), e] e(i I+D)^{-1} e+e
$$


so that $e(i I+D) e S-e \in \mathcal{K}$. By Lemma 2.1, it follows that $e(i I+D)^{-1} e=$ $(i e+e D e)^{-1}$ is a compact operator, that is, $\left(\operatorname{End}_{\mathcal{A}}(E), E \otimes_{\mathcal{A}} \mathscr{H}, D_{E, \nabla_{0}}\right)$ is a spectral triple. This along with the stability of spectral triples under inner fluctuations as proved above implies the result for arbitrary connections on $E$. Replacing the ideal of compact operators $\mathcal{K}$ by Schatten ideals we obtain the summability result.

\section{Universal families of spectral triples and determinant line bundles}

We follow a setup that is analogous to that of Freed [6], where it was done in the classical case of families of Dirac operators on a smooth manifold. This noncommutative geometry data will enable us to define the determinant line bundle associated to a natural family of spectral triples.

\subsection{Noncommutative geometry data for determinant line bundles:}

(1) a spectral triple, $(\mathcal{A}, \mathscr{H}, D)$;

(2) a Hermitian finite projective module $E$ over $\mathcal{A}$;

(3) a gauge group $\mathcal{E}$, which is a Lie subgroup of the group $\operatorname{Aut}_{\mathcal{A}}(E)$ of automorphisms of $E$ such that $\mathcal{E}$ acts smoothly and freely on $\mathcal{C}_{E}$ as follows: for $\xi \in E$, $\nabla \in \mathscr{C}_{E}$ and $g \in \mathcal{E}$, define $g \cdot \nabla(\xi):=\left(g^{-1} \otimes 1\right) \nabla(g \cdot \xi)$.

Remarks 3.1. 1 . There is an associated $\mathbb{Z}_{2}$-graded Hilbertian bundle $\mathcal{E} \otimes_{\mathcal{A}} \mathscr{H}$ over $\varphi_{E} / \mathscr{G}$

2. Therefore one obtains a family of spectral triples $\left(\operatorname{End}_{\mathcal{A}}(E), E \otimes_{\mathcal{A}} \mathscr{H}, D_{E, \nabla^{y}}\right)$, $y \in \mathscr{C}_{E} / \mathscr{E}$. For ease of notation, denote $D_{y}=D_{E, \nabla_{y}}$. Then $D$ can be viewed as an odd degree bundle map $D: \mathscr{E} \otimes_{\mathcal{A}} \mathscr{H} \rightarrow \mathcal{E} \otimes_{\mathcal{A}} \mathscr{H}$.

3. Under our assumptions, the quotient $\mathcal{C}_{E} / \mathscr{E}$ is a smooth manifold. If $B$ is a compact smooth finite dimensional submanifold of $\mathcal{C}_{E} / \mathscr{E}$, then all of the noncommutative data restrict to $B$.

4. In the case of the spectral triple for a spin manifold this is just the data for a family of twisted Dirac operators.

The construction of the determinant line bundle due to Quillen [12] is briefly adapted to our context in the remainder of the section. Consider the $\mathscr{E}$-equivariant family of spectral triples $\left\{\left(\operatorname{End}_{\mathcal{A}}(E), E \otimes_{\mathcal{A}} \mathscr{H}, D_{E, \nabla}\right) \mid \nabla \in \mathscr{C}_{E}\right\}$. The $\mathscr{\mathscr { C }}$-equivariant family of finite-dimensional spaces $\operatorname{ker} D_{E, \nabla}^{+} \subset E \otimes_{\mathcal{A}} \mathscr{H}^{+}$and $\operatorname{ker} D_{E, \nabla}^{-} \subset E \otimes_{\mathcal{A}}$ $\mathscr{H}^{-}$defines a virtual bundle $\operatorname{Index}\left(D_{\mathcal{E}, \nabla^{\mathcal{E}}}\right)$ over $B$, which is the index bundle for the family whose fibre at $\nabla$ is the virtual vector space $\operatorname{Index}\left(D_{\mathcal{E}, \nabla^{\mathcal{E}}}\right)_{\nabla}=\operatorname{ker} D_{E, \nabla}^{+}-$ $\operatorname{ker} D_{E, \nabla}^{-}$

Now $D_{E, \nabla}^{-} D_{E, \nabla}^{+}$and $D_{E, \nabla}^{+} D_{E, \nabla}^{-}$are self-adjoint operators, with discrete spectrum, and $\operatorname{spec}\left(D_{E, \nabla}^{-} D_{E, \nabla}^{+}\right)=\operatorname{spec}\left(D_{E, \nabla}^{+} D_{E, \nabla}^{-}\right)$. The simple argument to show 
this goes as follows. If $e$ is an eigenvector of $D_{E, \nabla}^{*} D_{E, \nabla}$ with eigenvalue $\lambda$, then $D_{E, \nabla} e$ is an eigenvector of $D_{E, \nabla} D_{E, \nabla}^{*}$ with eigenvalue $\lambda$. Also if $f$ is an eigenvector of $D_{E, \nabla} D_{E, \nabla}^{*}$ with eigenvalue $\eta$, then $D_{E, \nabla}^{*} f$ is an eigenvector of $D_{E, \nabla}^{*} D_{E, \nabla}$ with eigenvalue $\eta$. This shows that for $\lambda \neq 0, D_{E, \nabla}$ is an isomorphism from the $\lambda$-eigenspace of $D_{E, \nabla}^{*} D_{E, \nabla}$ to the $\lambda$-eigenspace of $D_{E, \nabla} D_{E, \nabla}^{*}$ (with inverse given by $\frac{1}{\lambda} D_{E, \nabla}^{*}$ ).

Let $H_{\nabla, \lambda}^{+}$denote the span of eigenvectors of $D_{E, \nabla}^{-} D_{E, \nabla}^{+}$with eigenvalue $<\lambda$, and $H_{\nabla, \lambda}^{-}$denote the span of eigenvectors of $D_{E, \nabla}^{+} D_{E, \nabla}^{-}$with eigenvalue $<\lambda$. These are smooth vector bundles over the open subset $U_{\lambda}=\{\nabla \in B \mid \lambda \notin$ $\left.\operatorname{spec}\left(D_{E, \nabla}^{-} D_{E, \nabla}^{+}\right)=\operatorname{spec}\left(D_{E, \nabla}^{+} D_{E, \nabla}^{-}\right)\right\}$.

It is easy to show that there is an exact sequence

$$
0 \rightarrow \operatorname{ker}\left(D_{E, \nabla}^{+}\right) \rightarrow H_{\nabla, \lambda}^{+} \rightarrow H_{\nabla, \lambda}^{-} \rightarrow \operatorname{ker}\left(D_{E, \nabla}^{-}\right) \rightarrow 0 .
$$

This gives rise to a canonical isomorphism

$$
\Lambda^{\max }\left(\operatorname{ker}\left(D_{E, \nabla}^{+}\right)^{*}\right) \otimes \Lambda^{\max }\left(\operatorname{ker}\left(D_{E, \nabla}^{-}\right)\right) \cong \Lambda^{\max }\left(H_{\nabla, \lambda}^{+}{ }^{*}\right) \otimes \Lambda^{\max }\left(H_{\nabla, \lambda}^{-}\right)
$$

Therefore we obtain a smooth line bundle $\mathscr{L}_{\lambda}:=\Lambda^{\max }\left(H_{\nabla, \lambda}^{+}{ }^{*}\right) \otimes \Lambda^{\max }\left(H_{\nabla, \lambda}^{-}\right)$over the open set $U_{\lambda}$. If $\mu>\lambda$, then $H_{\nabla, \mu}^{ \pm}=H_{\nabla, \lambda}^{ \pm} \oplus H_{\nabla, \lambda, \mu}^{ \pm}$, where $H_{\nabla, \lambda, \mu}^{+}$denotes the span of the eigenvectors of $D_{E, \nabla}^{-} D_{E, \nabla}^{+}$with eigenvalues lying in the open interval $(\lambda, \mu)$ and $H_{\nabla, \lambda, \mu}^{-}$denotes the span of the eigenvectors of $D_{E, \nabla}^{+} D_{E, \nabla}^{-}$with eigenvalues lying in the open interval $(\lambda, \mu)$. Therefore $\Lambda^{\max } H_{\nabla, \mu}^{ \pm} \cong \Lambda^{\max } H_{\nabla, \lambda}^{ \pm} \otimes$ $\Lambda^{\max } H_{\nabla, \lambda, \mu}^{ \pm}$. Since the restriction $\left.D_{E, \nabla}^{+}\right|_{H_{\nabla, \lambda, \mu}^{+}}: H_{\nabla, \lambda, \mu}^{+} \rightarrow H_{\nabla, \lambda, \mu}^{-}$is an isomorphism as observed earlier, we deduce that $\operatorname{det}\left(\left.D_{E, \nabla}^{+}\right|_{H_{\nabla, \lambda, \mu}^{+}}\right): \Lambda^{\max } H_{\nabla, \lambda, \mu}^{+} \rightarrow$ $\Lambda^{\max } H_{\nabla, \lambda, \mu}^{-}$is also an isomorphism. Therefore on the overlaps $U_{\lambda} \cap U_{\mu}$, there is a canonical identification of the determinant line bundles $\mathscr{L}_{\lambda}$ and $\mathscr{L}_{\mu}$ given by $s \mapsto s \otimes \operatorname{det}\left(\left.D_{E, \nabla}^{+}\right|_{H_{\nabla, \lambda, \mu}^{+}}\right)$. Since $\left\{U_{\lambda} \mid \lambda \in \mathbb{Q}\right\}$ is an open cover of $B$, we obtain a global determinant line bundle $\mathscr{L}$ over $B$ associated to the $\mathcal{G}$-equivariant family of spectral triples $\left\{\left(\mathcal{A}, E \otimes_{\mathcal{A}} \mathscr{H}, D_{E, \nabla}\right) \mid \nabla \in \mathcal{C}_{E}\right\}$. We state this as a proposition.

Proposition 3.2 (Determinant line bundle). Let ( $\mathcal{A}, \mathcal{H}, D)$ be a spectral triple satisfying the assumptions in Sec. 3.1, and $B$ a smooth compact submanifold of $\mathcal{C}_{E} / \mathscr{G}$. Then there is a smooth determinant line bundle $\mathscr{L}$ over $B$ associated to the $\mathcal{E}$-equivariant family of spectral triples $\left\{\left(\operatorname{End}_{\mathcal{A}}(E), E \otimes_{\mathcal{A}} \mathscr{H}, D_{E, \nabla}\right) \mid \nabla \in \mathcal{C}_{E}\right\}$, whose fibre at $\nabla \in B$ is naturally isomorphic to $\Lambda^{\max }\left(\operatorname{ker}\left(D_{E, \nabla}^{+}\right)^{*}\right) \otimes \Lambda^{\max }\left(\operatorname{ker}\left(D_{E, \nabla}^{-}\right)\right)$. 


\section{Stability of regular spectral triples, the Quillen metric and determinant section}

The Quillen metric on the determinant line bundle is obtained by patching metrics constructed on the open sets $U_{\lambda}$. As in the classical case this patching requires a zeta regularization of the metrics on $U_{\lambda}$. This in turn requires further assumptions on the spectral triple similar to the ones that Connes and Moscovici used. Let $\mathscr{H}^{\infty}=$ $\bigcap_{n \geq 1} \operatorname{Dom}|D|^{n}$; in this section we will assume that every $a \in \mathcal{A}$ maps $\mathscr{H}^{\infty}$ to itself.

Definition 4.1. 1. A spectral triple $(\mathcal{A}, \mathcal{H}, D)$ is said to be regular if $\mathcal{A}$ and $[D, \mathcal{A}]$ is contained in the domain of $\delta^{k}$ for all $k$. Here $\delta$ is the derivation $a \mapsto[|D|, a]$.

2. For a regular spectral triple $(\mathcal{A}, \mathscr{H}, D)$ let $\mathscr{B}_{0}$ denote the algebra generated by $\gamma, \operatorname{Sign}(D), \delta^{k}(a), \delta^{k}([D, a]), a \in \mathcal{A}, k \geq 0$. The dimension spectrum of a $p$-summable regular spectral triple $(\mathcal{A}, \mathscr{H}, D)$ is the smallest discrete subset $\Sigma \subset \mathbb{C}$ with the property that all the zeta functions

$$
\zeta(s, a)=\operatorname{Tr}\left(a|D+P|^{-s}\right), \quad a \in \mathscr{B}_{0}, s \in \mathbb{C}, \operatorname{Re}(s)>p
$$

have meromorphic continuations to $\mathbb{C}$ with poles contained in $\Sigma$, where $P$ denotes the orthogonal projection onto the nullspace of $D$.

$(\mathcal{A}, \mathscr{H}, D)$ is said to have simple dimension spectrum if the associated zeta functions $\zeta(s, a)$ have only simple poles, for all $a \in \mathscr{B}_{0}$.

Our next stability result is the following.

Proposition 4.2 (Stability of regular spectral triples). Let (A, $\mathcal{H}, D)$ be a regular spectral triple with simple dimension spectrum $\Sigma$, and let $E$ be a finite projective A-module with Hermitian structure and Hermitian connection $\nabla$. Then $\left(\operatorname{End}_{\mathcal{A}}(E), E \otimes_{\mathcal{A}} \mathscr{H}, D_{E, \nabla}\right)$ is also a regular spectral triple with simple dimension spectrum $\Sigma^{\prime}$ contained in $\Sigma-\mathbb{N}$. Moreover, if $0 \notin \Sigma$, then $0 \notin \Sigma^{\prime}$.

We defer the proof of this proposition to $\S 4.2$ and $\S 4.3$.

\subsection{Noncommutative geometry data for the Quillen metric and the determinant} section. We next modify the noncommutative geometry data given in Section 3.1, which will enable us to define the Quillen metric on the determinant line bundle and also to define the determinant section:

(1) a regular spectral triple, $(\mathcal{A}, \mathcal{H}, D)$ such that zero is not in the simple dimension spectrum;

(2) a Hermitian finite projective module $E$ over $\mathcal{A}$;

(3) a gauge group $\mathcal{E}$, which is a Lie subgroup of the group $\operatorname{Aut}_{\mathcal{A}}(E)$ of automorphisms of $E$ such that $\mathcal{G}$ acts smoothly and freely on $\mathcal{C}_{E}$ as follows: for $\xi \in E$, $\nabla \in \mathscr{C}_{E}$ and $g \in \mathcal{E}$, define $g \cdot \nabla(\xi):=\left(g^{-1} \otimes 1\right) \nabla(g \cdot \xi)$. 
To simplify notation, we will denote $\zeta(s):=\zeta(s, 1)$. Since by the hypotheses above 0 is not in the dimension spectrum $\Sigma$ of our regular spectral triple $(\mathcal{A}, \mathscr{H}, D)$, it enables us to define the derivative at zero $\zeta^{\prime}(0)$. In particular, the regularized determinant $\operatorname{det}\left(D^{*} D\right)=e^{-\zeta^{\prime}(0)}$ makes sense and is defined to be zero if $0 \in$ $\operatorname{spec}(D)$. Also, if $\zeta_{u}(s)=\operatorname{Tr}\left(\chi_{[u, \infty]}(|D|)\left|D^{\prime}\right|^{-s}\right)$ for $u>0$ and $u \notin \operatorname{spec}(|D|)$, where $\chi_{[u, \infty]}(|D|)$ denotes the spectral projection of $|D|$, then there is a simple relationship between the two zeta functions

$$
\zeta(s)=\sum_{0<\lambda<u} \frac{1}{\lambda^{s}}+\zeta_{u}(s), \quad \lambda \in \operatorname{spec}(|D|) .
$$

Since $\sum_{0<\lambda<u} \frac{1}{\lambda^{s}}, \lambda \in \operatorname{spec}(|D|)$, is an entire function, it follows that $\zeta_{u}(s)$ also has an analytic continuation to $\mathbb{C} \backslash \Sigma$, so that in particular the derivative at zero $\zeta_{u}^{\prime}(0)$ is defined. Moreover, Proposition 4.2, we see that the $\zeta^{\prime}(0, \nabla)$ and $\zeta_{u}^{\prime}(0, \nabla)$ are defined, where $\zeta_{u}(s, \nabla)=\operatorname{Tr}\left(\chi_{[u, \infty]}\left(\left|D_{E, \nabla}\right|\right)\left|D_{E, \nabla}^{\prime}\right|^{-s}\right)$ for $u \notin \operatorname{spec}\left(\left|D_{E, \nabla}\right|\right)$ and where $\chi[u, \infty]\left(\left|D_{E, \nabla}\right|\right)$ denotes the spectral projection of $\left|D_{E, \nabla}\right|$ and $\zeta(s, \nabla)=\zeta_{0}(s, \nabla)$.

With the geometric data as given above we will proceed to first construct the Quillen metric on the determinant line bundle $\mathscr{L}$. Using the notation of Section 3.1, we are given a Hermitian inner product on $E \otimes_{\mathcal{A}} \mathscr{H}$, which induces Hermitian metrics on the vector bundles $\mathscr{H}_{\lambda}$, since $\mathscr{H}_{\lambda, \nabla}$ is a finite dimensional subspace of the Hilbert space $E \otimes_{\mathcal{A}} \mathscr{H}$ for each $\nabla \in \mathcal{C}_{E}$. Let $g_{\mu}^{\prime}$ denote the induced Hermitian metric on the determinant line bundle $\mathscr{L}_{\lambda}$ over the open set $U_{\lambda}$. Due to the canonical identification of the determinant line bundles $\mathscr{L}_{\lambda}$ and $\mathscr{L}_{\mu}$ over $U_{\mu} \cap U_{\lambda}$ given by $s \mapsto s \otimes \operatorname{det}\left(\left.D_{E, \nabla}^{+}\right|_{H_{\nabla, \lambda, \mu}^{+}}\right)$, where $\mu>\lambda$, we see that

$$
g_{\mu}^{\prime}(s, s)=g_{\lambda}^{\prime}(s, s) \cdot\left(\prod_{\substack{\lambda<\tau<\mu \\ \tau \in \operatorname{spec}\left(D_{E}\right)}} \tau\right)^{2} .
$$

Therefore the induced Hermitian metric $g_{\lambda}^{\prime}$ does not define a smooth Hermitian metric on $\mathscr{L}$ but can, however, be modified as in [12] to give a smooth Hermitian metric $g$ on $\mathscr{L}$, called the Quillen metric, in the following way. At $\nabla \in U_{\lambda}$, define $g_{\lambda}=g_{\lambda}^{\prime} \cdot e^{-\zeta_{\lambda}^{\prime}(0, \nabla)}$. Then for $\nabla \in U_{\lambda} \cap U_{\mu}$, since

$$
e^{-\zeta_{\lambda}^{\prime}(0, \nabla)}=e^{-\zeta_{\mu}^{\prime}(0, \nabla)}\left(\prod_{\substack{\lambda<\tau<\mu \\ \tau \in \operatorname{spec}(D E, \nabla)}} \tau\right),
$$

we see that $g_{\lambda}=g_{\mu}$ on the overlap $U_{\lambda} \cap U_{\mu}$, showing that $g_{\mathscr{L}}:=g$ defines a smooth Hermitian metric on the determinant line bundle $\mathscr{L}$.

Proposition 4.3 (Quillen metric on the determinant line bundle). Let ( $\mathcal{A}, \mathcal{H}, D)$ be a regular spectral triple with dimension spectrum not containing zero. Let $B$ be a compact submanifold of $\mathcal{C}_{E} / \mathcal{E}$. Then there is a smooth Hermitian metric $g_{\mathscr{L}}$ (the Quillen metric) on the determinant line bundle $\mathscr{L}$ over $B$ associated to the $\mathcal{G}$-equivariant family of spectral triples $\left\{\left(\operatorname{End}_{\mathcal{A}}(E), E \otimes_{\mathcal{A}} \mathcal{H}, D_{E, \nabla}\right) \mid \nabla \in \mathcal{C}_{E}\right\}$. 
We next describe the determinant section of $\mathscr{L}$ over the open set $U_{\lambda} \cap U_{0}$. Let $\left\{\psi_{1}, \ldots, \psi_{n}\right\}$ be a basis of eigenvectors in $\mathscr{H}_{\lambda}^{+}$and let $\left\{\psi_{1}^{*}, \ldots, \psi_{n}^{*}\right\}$ be the dual basis. Then the determinant section is $\operatorname{det}\left(D_{E, \nabla, \lambda}^{+}\right)=\left(\psi_{1}^{*} \wedge \cdots \wedge \psi_{N}^{*}\right) \otimes\left(D \psi_{1} \wedge \cdots \wedge D \psi_{N}\right)$. On the overlap $U_{\lambda} \cap U_{\mu} \cap U_{0}$, it is easy to see that

$$
\operatorname{det}\left(D_{E, \nabla, \mu}^{+}\right)=\operatorname{det}\left(D_{E, \nabla, \lambda}^{+}\right)\left(\prod_{\substack{\lambda<\tau<\mu \\ \tau \in \operatorname{spec}\left(D_{E}\right)}} \tau\right) .
$$

It follows that on the open set $U_{0}$ there is a smooth section $\operatorname{det}\left(D_{E}\right)$ of the determinant line bundle $\mathscr{L}$.

Proposition 4.4 (Determinant section of the determinant line bundle). Let $(\mathcal{A}, \mathscr{H}, D)$ be a regular spectral triple with dimension spectrum not containing zero. Let $B$ be a compact submanifold of $\mathcal{C}_{E} / \mathscr{G}$. Then there is a smooth determinant section $\operatorname{det}\left(D_{E}\right)$ of the determinant line bundle $\mathscr{L}$ over $B \cap U_{0}$ associated to the $\mathcal{G}$-equivariant family of spectral triples $\left\{\left(\operatorname{End}_{\mathcal{A}}(E), E \otimes_{\mathcal{A}} \mathscr{H}, D_{E, \nabla}\right) \mid \nabla \in \mathscr{C}_{E}\right\}$.

4.2. Proof of Proposition 4.2. We begin by proving the following special case of Proposition 4.2.

Proposition 4.5 (Stability of regular spectral triples). Let $(\mathcal{A}, \mathcal{H}, D)$ be a regular spectral triple with simple dimension spectrum $\Sigma$, let $E$ be a finite projective A-module with Hermitian structure and Hermitian connection $\nabla$. In addition, we assume that $0 \notin \operatorname{spec}(D)$ and $0 \notin \operatorname{spec}\left(D_{E, \nabla}\right)$. Then $\left(\operatorname{End}_{\mathcal{A}}(E), E \otimes_{\mathcal{A}} \mathcal{H}, D_{E, \nabla}\right)$ is also a regular spectral triple with simple dimension spectrum $\Sigma^{\prime}$ contained in $\Sigma-\mathbb{N}$. Moreover, if $0 \notin \Sigma$ then $0 \notin \Sigma^{\prime}$.

To prove regularity we will use Higson's characterization of regularity. For that we recall the following definition.

Definition 4.6. Let $(\mathcal{A}, \mathscr{H}, D)$ be a spectral triple such that $\mathscr{A}$ maps $\mathscr{H}^{\infty}$ to itself. The algebra of differential operators $\mathscr{D}(\mathcal{A}, \Delta)$ is the smallest algebra of operators on $\mathscr{H}^{\infty}$ closed under the operation $T \mapsto[\Delta, T]$ containing $\mathcal{A}$ and $[D, \mathcal{A}]$. Here $\Delta$ denotes $D^{2}$.

This algebra is filtered as follows. The elements of $\mathcal{A}$ and $[D, \mathcal{A}]$ have order zero and the operation $[\Delta, \cdot]$ raises order at most by one. Thus $\mathscr{D}_{k}$, the space of operators of order at most $k$, is defined inductively as follows:

$\mathscr{D}_{0}=$ algebra generated by $\mathcal{A}$ and $[D, \mathcal{A}]$,

$\mathscr{D}_{1}=\mathscr{D}_{0}+\left[\Delta, \mathscr{D}_{0}\right]+\mathscr{D}_{0}\left[\Delta, \mathscr{D}_{0}\right]$,

$\mathscr{D}_{k}=\mathscr{D}_{0}+\left[\Delta, \mathscr{D}_{k-1}\right]+\mathscr{D}_{0}\left[\Delta, \mathscr{D}_{k-1}\right]+\sum_{j=1}^{k-1} \mathscr{D}_{j} \mathscr{D}_{k-j}$.

Definition 4.7. A spectral triple $(\mathcal{A}, \mathcal{H}, D)$ satisfies the basic estimate if for every differential operator $X \in \mathscr{D}_{k}$ there is an $\epsilon>0$ such that for all $v \in \mathscr{H}^{\infty}$,

$$
\left\|D^{k} v\right\|+\|v\| \geq \epsilon\|X v\| \text {. }
$$


Theorem 4.8 (Higson, Theorem 4.26, [9]). Let ( $(\mathcal{A}, \mathcal{H}, D)$ be a spectral triple such that every a $\in \mathcal{A}$ maps $\mathscr{H}^{\infty}$ to itself. Then this spectral triple is regular iff it satisfies the basic estimate.

Proof of Proposition 4.5. For notational convenience let us denote $D_{E, \nabla}$ by $D^{\prime}$. Let $\Delta^{\prime}=D^{\prime 2}$ and $\mathscr{D}\left(\operatorname{End}_{\mathcal{A}}(E), \Delta^{\prime}\right)$ be the associated differential algebra with the filtration $\mathscr{D}^{\prime}$. Then note that $\mathscr{D}_{k}^{\prime} \subseteq \mathscr{D}_{k}$. Therefore $\left(\operatorname{End}_{\mathcal{A}}(E), E \otimes_{\mathcal{A}} \mathscr{H}, D^{\prime}\right)$ satisfies the basic estimate hence regularity.

Now we proceed to the stability of the dimension spectrum. This is the final part of Proposition 4.5. We will divide this in two cases. First let us tackle the case of free modules. Then if necessary by considering matrices with entries from $\mathcal{A}$ we may assume that $E=\mathcal{A}$.

Lemma 4.9. Let $T \in \mathscr{B}$ and $0<\operatorname{Re}(\lambda)<\min \{d \in \sigma(\Delta)\}$. Then:

(a) $(\lambda-\Delta)^{-1} T=\sum_{k=1}^{n} \sum_{j=0}^{k} T_{j k}|D|^{j}(\lambda-\Delta)^{-1-k}+R_{n}(\lambda, T)$ where $T_{j k}=$ $\left(\begin{array}{l}k \\ j\end{array}\right) 2^{j} \delta^{2 k-j}(T)$.

(b) $\left\|R_{n}(\lambda, T)\right\|_{1}<C \max (|\operatorname{Im} \lambda|, 1)^{-n / 2}$.

(c) The function $z \mapsto \int_{C-i \infty}^{C+i \infty} \lambda^{-z} R_{n}(\lambda, T) D(\lambda-\Delta)^{-1} d \lambda$ is holomorphic for large enough $n$ where $C$ is a real number separating zero from the spectrum of $\Delta$.

Proof. (a) To prove (a) note that

$$
\begin{aligned}
(\lambda-\Delta)^{-1} T & =T(\lambda-\Delta)^{-1}+\left[(\lambda-\Delta)^{-1}, T\right] \\
& =T(\lambda-\Delta)^{-1}+(\lambda-\Delta)^{-1}[\Delta, T](\lambda-\Delta)^{-1} \\
& =\sum_{0 \leq k \leq n} T^{(k)}(\lambda-\Delta)^{-1-k}+(\lambda-\Delta)^{-1} T^{(n+1)}(\lambda-\Delta)^{-n} .
\end{aligned}
$$

Here the last equality is obtained by iterating the previous one $k$ times, and the $T^{(k)}$ 's are defined inductively by $T^{(0)}=T$ and $T^{(k)}=\left[\Delta, T^{(k-1)}\right]$. For $b \in \mathscr{B}$ we also have the following relations:

$$
\begin{aligned}
{[\Delta, b] } & =|D| \delta(b)+\delta(b)|D|, \\
|D| b & =b|D|+\delta(b) .
\end{aligned}
$$

Combining these two we get for $T \in \mathscr{B}$,

$$
T^{(k)}=2 \delta\left(T^{(k-1)}\right)|D|+\delta^{2}\left(T^{(k-1)}\right) .
$$

Applying this repeatedly we obtain that

$$
T^{(k)}=\sum_{0 \leq j \leq k}\left(\begin{array}{c}
k \\
j
\end{array}\right) 2^{j} \delta^{2 k-j}(T)|D|^{j}=T_{j k}|D|^{j} .
$$


(b) To prove (b) note that

$$
\begin{aligned}
\left\||D|(\lambda-\Delta)^{-1 / 2}\right\| & \leq \sup _{d \in \sigma(|D|)} \frac{d}{\left|\lambda-d^{2}\right|} \\
& =\sup _{d \in \sigma(|D|)} \sqrt{\frac{d^{2}}{\left(\operatorname{Re}(\lambda)-d^{2}\right)^{2}+\operatorname{Im}(\lambda)^{2}}} \\
& \leq \sup _{d \in \sigma(|D|)} \sqrt{\frac{d^{2}}{\left(\operatorname{Im}(\lambda)^{2}\left(\operatorname{Re}(\lambda)-d^{2}\right)^{2}\right)}} \text { if } \operatorname{Im}(\lambda)>1 \\
& \leq \frac{1}{|\operatorname{Re}(\lambda)|^{1 / 4} \mid(1-\sqrt{\operatorname{Re}(\lambda) \mid}}
\end{aligned}
$$

and is less than or equal to a constant when $\operatorname{Im}(\lambda) \leq 1$. Also, for the trace norm we have

$$
\left\|(\lambda-\Delta)^{-\alpha}\right\|_{1} \leq \frac{C}{\max (|\operatorname{Im}(\lambda)|, 1)^{\alpha / 2}} .
$$

Therefore $\left\||D|(\lambda-\Delta)^{-1 / 2}\right\| \leq \frac{C}{\max (|\operatorname{Im}(\lambda)|, 1)}$. Hence, for $j \leq n$ one gets the trace norm estimate

$$
\begin{aligned}
\left\||D|^{j}(\lambda-\Delta)^{-n}\right\|_{1} & \leq\left\||D|^{j}(\lambda-\Delta)^{-j / 2}\right\|\left\|(\lambda-\Delta)^{-(n-j / 2)}\right\|_{1} \\
& \leq \frac{C_{1}}{\max (|\operatorname{Im}(\lambda)|, 1)^{n / 2}} .
\end{aligned}
$$

Therefore the trace norm estimate for the remainder follows.

(c) The proof of (c) immediately follows from part (b).

Let $D^{\prime}=D+B$ for some $B \in \mathscr{B}$. Then $\Delta^{\prime}=(D+B)^{2}=D^{2}+R=\Delta+R$, where $R=D B+B D+B^{2}$. By the resolvent identity one has

$$
\begin{aligned}
\left(\lambda-\Delta^{\prime}\right)^{-1} & =\left(1-(\lambda-\Delta)^{-1} R\right)^{-1}(\lambda-\Delta)^{-1} \\
& =(1-X)^{-1}(\lambda-\Delta)^{-1} \\
& =\sum_{0 \leq k \leq n} X^{k}(\lambda-\Delta)^{-1}+X^{n+1}(1-X)^{-1}(\lambda-\Delta)^{-1},
\end{aligned}
$$

where $X=(\lambda-\Delta)^{-1} R$ and we have used the identity

$$
(1-X)^{-1}=\sum_{k \leq n} X^{k}+X^{n+1}(1-X)^{-1} .
$$

By hypothesis, for $b \in \mathscr{B}$, the function

$$
\operatorname{Tr} b|D|^{-2 s}: s \rightarrow \operatorname{Tr}\left(b \int_{C-i \infty}^{C+i \infty} \lambda^{-s}(\lambda-\Delta)^{-1} d \lambda\right)
$$


is well defined on the half plane $\operatorname{Re}(s)>p$, is analytic there, and has a meromorphic continuation to $\mathbb{C}$ with poles contained in $\Sigma$. Here $C$ is a real number separating zero from the spectrum of $\Delta$ and $\Delta^{\prime}$. We want to prove the same is true when $\Delta$ is replaced by $\Delta^{\prime}$ and that is achieved by analyzing the meromorphic continuation of the difference $\operatorname{Tr} b\left|D^{\prime}\right|^{-2 z}-\operatorname{Tr} b|D|^{-2 z}$. For that observe that by (2),

$$
\begin{aligned}
\operatorname{Tr} & b\left|D^{\prime}\right|^{-2 z}-\operatorname{Tr} b|D|^{-2 z} \\
& =\operatorname{Tr} b\left(\int_{C-i \infty}^{C+i \infty} \lambda^{-z}\left(\left(\lambda-\Delta^{\prime}\right)^{-1}-(\lambda-\Delta)^{-1}\right) d \lambda\right) \\
& =\operatorname{Tr} b\left(\int_{C-i \infty}^{C+i \infty} \lambda^{-z}\left(\sum_{1 \leq k \leq n} X^{k}(\lambda-\Delta)^{-1}+X^{n+1}(1-X)^{-1}(\lambda-\Delta)^{-1}\right) d \lambda\right)
\end{aligned}
$$

Lemma 4.10. On any given right half plane, for large enough $n$ the function

$$
z \mapsto \operatorname{Tr}\left(b \int_{C-i \infty}^{C+i \infty} \lambda^{-z} X^{n+1}(1-X)^{-1}(\lambda-\Delta)^{-1} d \lambda\right)
$$

is holomorphic.

Proof. For a compact operator $T$ let $\mu_{n}(T)$ be the $n$th largest singular value of $T$. Let $d_{n}=\mu_{n}\left(D^{-1}\right)$. Then we have the following bounds

$$
\mu_{n}(X)<C d_{n}, \quad\|X\|=\mu_{1}(X)<C(|y|+1)^{-1},
$$

where $y=\operatorname{Im}(\lambda)$. We know $\sum d_{n}^{p+1}$ is finite. Therefore by Hölder's inequality it follows that, for $n$ large enough, the trace norm of $X^{n+1}(1-X)^{-1}(\lambda-\Delta)^{-1}$ is bounded by $(|\operatorname{Im}(\lambda)|+1)^{-k}$, which implies the result.

Lemma 4.11. For every $k \geq 1$,

$$
z \mapsto \operatorname{Tr} b\left(\int_{C-i \infty}^{C+i \infty} \lambda^{-z} X^{k}(\lambda-\Delta)^{-1} d \lambda\right)
$$

defines a meromorphic function on $\mathbb{C}$ with poles contained in the set $\Sigma-\mathbb{N}:=\{s-n \mid$ $s \in \Sigma, n \geq 0\}$. Furthermore, this function is regular at zero.

Proof. We will prove it for $k=1$, in the general case the proof is similar. In this case,

$$
\begin{aligned}
& \operatorname{Tr} b\left(\int_{C-i \infty}^{C+i \infty} \lambda^{-z} X(\lambda-\Delta)^{-1} d \lambda\right) \\
&= \operatorname{Tr} b\left(\int_{C-i \infty}^{C+i \infty} \lambda^{-z}(\lambda-\Delta)^{-1} D B(\lambda-\Delta)^{-1} d \lambda\right) \\
& \quad+\operatorname{Tr} b\left(\int_{C-i \infty}^{C+i \infty} \lambda^{-z}(\lambda-\Delta)^{-1} B D(\lambda-\Delta)^{-1} d \lambda\right) \\
&+\operatorname{Tr} b\left(\int_{C-i \infty}^{C+i \infty} \lambda^{-z}(\lambda-\Delta)^{-1} B^{2}(\lambda-\Delta)^{-1} d \lambda\right) .
\end{aligned}
$$


We want to show that each term admits a meromorphic continuation to the complex plane with poles in $\Sigma-\mathbb{N}$. The first two terms are similar and we will only tackle the second term. From the analysis for the second term the result for the third term will follow. By Lemma 4.9,

$$
\begin{aligned}
\operatorname{Tr} b( & \left.\int_{C-i \infty}^{C+i \infty} \lambda^{-z}(\lambda-\Delta)^{-1} B D(\lambda-\Delta)^{-1} d \lambda\right) \\
= & \sum_{0 \leq k \leq n} \sum_{0 \leq j \leq k} \operatorname{Tr} b\left(\int_{C-i \infty}^{C+i \infty} \lambda^{-z} B_{j k}|D|^{j} D(\lambda-\Delta)^{-2-k} d \lambda\right) \\
& \quad+\operatorname{Tr} b\left(\int_{C-i \infty}^{C+i \infty} \lambda^{-z} R_{n}(\lambda, B) D(\lambda-\Delta)^{-1} d \lambda\right) \\
= & \sum_{0 \leq k \leq n} \operatorname{Tr} b \sum_{0 \leq j \leq k} B_{j k} \operatorname{Sign}(D)\left(\begin{array}{c}
-z \\
k+1
\end{array}\right)|D|^{-z-k+j} \\
& + \text { a holomorphic function on a right half plane } \\
= & \sum_{0 \leq k \leq n}\left(\begin{array}{c}
-z \\
k+1
\end{array}\right) \sum_{0 \leq j \leq k} \zeta(z+k-j, b) \sum_{0 \leq j \leq k} B_{j k} \operatorname{Sign}(D) \\
& \quad+\text { a holomorphic function on a right half plane, }
\end{aligned}
$$

where the first equality follows from Lemma 4.9 (a). Clearly poles of this function are contained in $\Sigma-\mathbb{N}$. The poles of this function are simple provided the zeta functions involved have simple poles. Note that if we assume that $\zeta(z, b)$ has simple poles for all $b \in \mathscr{B}$ then we also get the regularity at zero.

These lemmas give an alternative proof of the following result in [3].

Corollary 4.12. Let $(\mathcal{A}, \mathscr{H}, D)$ be a regular spectral triple with simple dimension spectrum $\Sigma$. Suppose that $0 \notin \operatorname{spec}(D)$ and $0 \notin \operatorname{spec}(D+T)$ for $T \in \mathscr{B}_{0}$. Then $(\mathcal{A}, \mathcal{H}, D+T)$ is also a regular spectral triple with simple dimension spectrum $\Sigma^{\prime}$ contained in $\Sigma-\mathbb{N}$. Moreover, if $0 \notin \Sigma$ then $0 \notin \Sigma^{\prime}$.

This proves Proposition 4.5 for the case when $E$ is a finite free module. Let $E=$ $p \mathcal{A}$, where $p$ is a projection in $\mathcal{A}$, and $q=(1-p)$. Then $T=D-p D p-q D q \in \mathscr{B}_{0}$ and $D+T=p D p+q D q$. By the corollary above, we know that $(\mathcal{A}, \mathcal{H}, p D p+q D q)$ is also a regular spectral triple with simple dimension spectrum $\Sigma^{\prime}$ contained in $\Sigma-\mathbb{N}$. Now

$$
\zeta(z, b)=\operatorname{Tr}\left(p b p|p D p+q D q|^{-z}\right)=\operatorname{Tr}\left(p b p|p D p|^{-z}\right),
$$

since $p q=0$. Therefore, by Corollary 4.12, $(p \mathcal{A} p, p \mathscr{H}, p D p)$ is also a regular spectral triple with simple dimension spectrum $\Sigma^{\prime}$ contained in $\Sigma-\mathbb{N}$, where we observe that $\operatorname{End}_{\mathcal{A}}(E)=p \mathcal{A} p$. This completes the proof of Proposition 4.5.

4.3. Removing the hypothesis "zero not in the spectrum". We will now remove the hypotheses $0 \notin \operatorname{spec}(D)$ and $0 \notin \operatorname{spec}(D+T)$ in Corollary 4.12. The difficulty lies in the fact that the orthogonal projection onto the nullspace of $D+T$, namely 
$P_{0}(D+T)$, is not in any obvious way in the algebra $\mathscr{B}_{0}$ whenever $T \in \mathscr{B}_{0}$. So we will suitably enlarge the algebra $\mathscr{B}_{0}$ to accommodate these projections in such a way that the enlarged algebra does not alter the meromorphic continuation properties of the zeta functions.

Define the space of all smoothing operators

$$
\Psi^{-\infty}:=\left\{\left.a \in B(\mathscr{H})|| D\right|^{k} a|D|^{l} \text { is bounded for all } k, l \geq 0\right\} .
$$

Then $\Psi^{-\infty}$ is an ideal in the algebra $\left\{a \in B(\mathscr{H}) \mid a\left(\mathscr{H}^{\infty}\right) \subset \mathscr{H}^{\infty}\right.$ and $a \in$ $\left.\bigcap_{n \geq 1} \operatorname{Dom} \delta^{n}\right\}$.

Definition 4.13. Let $\mathscr{B}$ denote the algebra generated by $\mathscr{B}_{0}$ and $\Psi^{-\infty}$.

Proposition 4.14. The zeta functions $z \mapsto \zeta(z, b)=\operatorname{Tr}\left(b\left|D+P_{0}(D)\right|^{-z}\right), b \in \mathscr{B}$ have the same meromorphic continuation properties as that of $z \mapsto \zeta(z, b), b \in \mathscr{B}_{0}$.

Proof. We begin by proving that $z \mapsto \zeta(z, b)$ is an entire function for $b \in \Psi^{-\infty}$. This follows from the fact that $\zeta(z, b)=\operatorname{Tr}\left(b\left|D+P_{0}(D)\right|^{k}\left|D+P_{0}(D)\right|^{-(k+z)}\right)$ is holomorphic for $\operatorname{Re}(z)>p-k$ for all $k \geq 0$. Here we have also used that $b\left|D+P_{0}(D)\right|^{k}$ is bounded whenever $b|D|^{k}$ is bounded for all $k \geq 0$.

Notice that $\Psi^{-\infty}$ is an ideal in $\mathscr{B}$, therefore the proposition follows.

Lemma 4.15. The projections onto the nullspace of the operators of the form $D+T$ are smoothing operators for all $T \in \mathscr{B}_{0}$.

Proof. Since $P_{0}(D)$ is smoothing and $D^{m} P_{0}(D)=0$. To show that $P_{0}(D+T)$ is smoothing it is enough to show that $D^{m}\left(P_{0}(D+T)-P_{0}(D)\right) D^{l}$ is bounded for all $m, l \geq 0$. Let $D^{\prime}=D+T$ for some $T \in \mathcal{B}$. Then $\Delta^{\prime}=(D+T)^{2}=D^{2}+R=$ $\Delta+R$, where $R=D T+T D+T^{2}$. Note that by the Cauchy formula we have

$$
\begin{aligned}
D^{m} & \left(P_{0}\left(D^{\prime}\right)-P_{0}(D)\right) D^{l} \\
& =\oint_{C_{r}} d \lambda D^{m}\left(\left(\lambda-\Delta^{\prime}\right)^{-1}-(\lambda-\Delta)^{-1}\right) D^{l} \\
& =\oint_{C_{r}} d \lambda D^{m}\left(\sum_{1 \leq k \leq n} X^{k}(\lambda-\Delta)^{-1}+X^{n+1}(1-X)^{-1}(\lambda-\Delta)^{-1}\right) D^{l},
\end{aligned}
$$

where $X=(\lambda-\Delta)^{-1} R$ and we have used the identity

$$
(1-X)^{-1}=\sum_{k \leq n} X^{k}+X^{n+1}(1-X)^{-1} .
$$

Here $\mathcal{C}_{r}$ is a circle of radius $r$ centered at the origin in $\mathbb{C}$, where $r>$ is small.

We analyse the expression when $k=1$ as well as the remainder term (the other terms can be analysed similarly):

$$
\oint_{\mathcal{C}_{r}} d \lambda D^{m}(\lambda-\Delta)^{-1}\left(D T+T D+T^{2}\right)(\lambda-\Delta)^{-1} D^{l}
$$


is equal to

$$
\oint_{\mathcal{C}_{r}} d \lambda D^{m}(\lambda-\Delta)^{-1}\left([D, T]+2 T D+T^{2}\right)(\lambda-\Delta)^{-1} D^{l} .
$$

We analyse the $T D$-term

$$
\oint_{\mathcal{C}_{r}} d \lambda D^{m}(\lambda-\Delta)^{-1} T D(\lambda-\Delta)^{-1} D^{l}
$$

Applying part (a) of Lemma 4.9, we obtain

$$
\begin{aligned}
\sum_{0 \leq k \leq n} \sum_{0 \leq j \leq k} \oint_{e_{r}} & D^{m} T_{j k}|D|^{j} D(\lambda-\Delta)^{-2-k} D^{l} d \lambda \\
& +\oint_{e_{r}} D^{m} R_{n}(\lambda, B) D(\lambda-\Delta)^{-1} D^{l} d \lambda
\end{aligned}
$$

Upon commuting $D^{m}$ and $T_{j k}$, at the expense of adding more commutator terms, we get terms of the form

$$
T_{j k} \oint_{e_{r}} D^{m+l+j+1} \operatorname{Sign}(D)(\lambda-\Delta)^{-2-k} d \lambda,
$$

which clearly vanish, together with a remainder term

$$
\oint_{\mathcal{C}_{r}} D^{m} R_{n}(\lambda, B) D(\lambda-\Delta)^{-1} D^{l} d \lambda .
$$

The remainder is bounded since it contains $(\lambda-\Delta)^{-1-n}$, which dominates $D^{m}$ whenever $m+l \leq n+1$.

Lemma 4.16. The projections onto the nullspace of the operators $D_{E, \nabla}$ are smoothing operators.

Proof. Let $E=p \mathcal{A}$, where $p$ is a projection in $\mathcal{A}$ and let $\nabla_{0}=p d p$ be the Grassmanian connection. We need to show that the projection onto the nullspace of $D_{E, \nabla_{0}}=p D p$ is a smoothing operator. If $q=(1-p)$, then $T=p D p+q D q-D \in$ $\mathscr{B}_{0}$. It follows that $p D p+q D q$ is an operator of the form as in Lemma 4.15. Therefore the projection onto the nullspace of $p D p+q D q$ is a smoothing operator. But $P_{0}(p D p)$ is just $p P_{0}(D)$, which is smoothing since $p \in \mathscr{B}_{0}$ and smoothing operators form an ideal. A general connection $\nabla$ on $E$ is of the form $\nabla_{0}+T$ and so $D_{E, \nabla}$ is of the form $D_{E, \nabla_{0}}+T$. Applying Lemma 4.15, we deduce the lemma.

Proposition 4.14 and Lemmas 4.15, 4.16 imply Proposition 4.2, which is just Proposition 4.5 with the hypotheses "zero not in the spectrum" removed. 


\section{An example}

Here we study the case of the noncommutative torus, for which the family of spectral triples is regular, and therefore the determinant line bundle $\mathscr{L}$ has a Quillen metric and determinant section over the moduli space of flat rank 1 connections, which we calculate explicitly in terms of theta and eta functions on the moduli space which is a torus.

Recall that the noncommutative torus $A_{\theta}$ is the universal $\mathrm{C}^{*}$-algebra generated by two unitaries $U, V$ satisfying the Weyl commutation relations $U V=e^{i \theta} V U$ for fixed $\theta \in \mathbb{R}$. There is a natural smooth subalgebra $A_{\theta}^{\infty}$ called the smooth noncommutative torus, which is defined as those elements in $A_{\theta}$ that can be represented by infinite power series $f=\sum_{(m, n) \in \mathbb{Z}^{2}} a_{(n, m)} U^{m} V^{n}$, with $\left(a_{(m, n)}\right) \in S\left(\mathbb{Z}^{2}\right)$, the Schwartz space of rapidly decreasing sequences on $\mathbb{Z}^{2}$.

We next briefly recall the space of differential forms on $A_{\theta}^{\infty}$, which is defined by $\Omega^{j}\left(A_{\theta}^{\infty}\right)=A_{\theta}^{\infty} \otimes \Lambda^{j}\left(\mathbb{C}^{2}\right)$. We remark that there are two notions of differential forms ([2], [5]) and in the context of the noncommutative torus one knows [7] through explicit computations that they agree. The differential $d$ is defined as $d(a \otimes \alpha):=$ $\delta_{1}(a) \otimes e_{1} \wedge \alpha+\delta_{2}(a) \otimes e_{2} \wedge \alpha$ where $a \in A_{\theta}^{\infty}, \alpha \in \Lambda^{\bullet}\left(\mathbb{C}^{2}\right)$, and $e_{j}, j=1,2$, is the canonical basis of $\mathbb{C}^{2}$. Here the standard derivations are defined on the generators by $\delta_{1}(U)=2 \pi U, \delta_{2}(V)=2 \pi V, \delta_{1}(V)=\delta_{2}(U)=0$.

We now consider the space of all flat Hermitian connections on the trivial rank one free module over $A_{\theta}^{\infty}$. The space of all such connections is an affine space $\ell_{A_{\theta}^{\infty}}$ with associated vector space $Z^{1}\left(A_{\theta}^{\infty}\right)$, which is the vector space of closed 1-forms on $A_{\theta}^{\infty}$. Let $U\left(A_{\theta}^{\infty}\right)$ denote the space of all unitary elements in $A_{\theta}^{\infty}$. Then by [5], Proposition 5.7, we know that the quotient space $\ell_{A_{\theta}^{\infty}} / U\left(A_{\theta}^{\infty}\right)$ is homeomorphic to the torus $\mathbb{T}^{2}$. This is the analogue of the Jacobian variety for the noncommutative torus $A_{\theta}^{\infty}$.

We next recall the spectral triple on $A_{\theta}^{\infty}$; cf. [8], Section 4. Let $\mathscr{H}=\mathscr{H}^{+} \oplus \mathscr{H}^{-}$, where $\mathscr{H}^{ \pm}$are both copies of $L^{2}\left(A_{\theta}\right.$, tr) with tr the canonical trace on $A_{\theta}$. Fix $\tau \in \mathbb{C}$ such that $\operatorname{Im}(\tau)>0$, and define $\partial=\partial_{\tau}=\delta_{1}+\tau \delta_{2}$ so that $\partial^{*}=-\delta_{1}-\bar{\tau} \delta_{2}$. Finally, let $D=\left(\begin{array}{cc}0 & \partial^{*} \\ \partial & 0\end{array}\right)$. Then a spectral triple for $A_{\theta}^{\infty}$ is $\left(A_{\theta}^{\infty}, \mathcal{H}, D\right)$. From what follows, this is a regular spectral triple.

The flat Hermitian connections are $\nabla=\nabla_{\tau}=\left(\delta_{1}+u\right) \otimes e_{1}+\tau\left(\delta_{2}+v\right) \otimes e_{2}$, where $u, v \in[0,1)$, being thought of as parametrizing the Jacobian variety. Then setting $\partial_{\nabla}=\left(\delta_{1}+u\right)+\tau\left(\delta_{2}+v\right)$, we see that $\partial_{\nabla}^{*}=-\left(\delta_{1}+u\right)-\bar{\tau}\left(\delta_{2}+v\right)$ and $D_{\nabla}=\left(\begin{array}{cc}0 & \partial_{\nabla}^{*} \\ \partial_{\nabla} & 0\end{array}\right)$. Moreover, $D^{2}=\left(\begin{array}{cc}\partial_{\nabla}^{*} \partial_{\nabla} & 0 \\ 0 & \partial_{\nabla} \partial_{\nabla}^{*}\end{array}\right)$ and the vectors $U^{m} V^{n}$ form an orthonormal basis of eigenvectors for both $\partial_{\nabla}^{*} \partial_{\nabla}$ and $\partial_{\nabla} \partial_{\nabla}^{*}$. We compute the eigenvalues

$$
\begin{aligned}
\partial_{\nabla}^{*} \partial_{\nabla}\left(U^{m} V^{n}\right) & =\partial_{\nabla} \partial_{\nabla}^{*}\left(U^{m} V^{n}\right) \\
& =-\left(\left(\delta_{1}+u\right)+\tau\left(\delta_{2}+v\right)\right)\left(\left(\delta_{1}+u\right)+\bar{\tau}\left(\delta_{2}+v\right)\right)\left(U^{m} V^{n}\right)
\end{aligned}
$$


The geometry of determinant line bundles in noncommutative geometry

$$
=-\frac{4 \pi^{2}}{\operatorname{Im}(\tau)^{2}}|(m+u)-\tau(n+v)|^{2} .
$$

The associated zeta function

$$
\zeta(s)=\sum_{m, n} \frac{\operatorname{Im}(\tau)^{2 s}}{4^{s} \pi^{2 s}}|(m+u)-\tau(n+v)|^{-2 s}
$$

is holomorphic for $\operatorname{Re}(s) \gg 0$ and is precisely the $\zeta$-function considered in the proof of Theorem 4.1 of [13], where it arose in a completely different context. Using the results from there, it follows that $\zeta(s)$ has a meromorphic continuation to $\mathbb{C}$, with no pole at $s=0$, showing in particular that the spectral triple that we started out with on the noncommutative torus is a regular spectral triple such that zero is not in the dimension spectrum. Moreover, the Quillen norm of the determinant section of the determinant line bundle $\mathscr{L}$ over the Jacobian variety is

$$
\mathbb{T}^{2} \ni(u, v) \mapsto\|\operatorname{det}(D)\|_{\mathscr{L}}(u, v)=e^{-\zeta^{\prime}(0)}=\left|e^{\pi i v^{2} \tau} \frac{\theta_{1}(u-\tau v, \tau)}{\eta(\tau)}\right| \in \mathbb{R}^{\geq 0},
$$

where the theta function is defined as

$$
\theta_{1}(w, \tau)=-\eta(\tau) e^{\pi i(w+\tau / 6)} \prod_{k=-\infty}^{\infty}\left(1-e^{2 \pi i\left(|k| \tau-\epsilon_{k} w\right)},\right.
$$

where $\epsilon_{k}=\operatorname{Sign}\left(k+\frac{1}{2}\right)$, and the Dedekind eta function is defined by

$$
\eta(\tau)=e^{\pi i \tau / 12} \prod_{k=1}^{\infty}\left(1-e^{2 \pi i k \tau}\right) .
$$

From these formulae, one immediately recovers the determinant section as in the text of the paper. Also by the explicit knowledge of the eigenvalues and the determinant,

$$
e^{-\zeta_{\lambda}^{\prime}(0)}=\left|e^{\pi i v^{2} \tau} \frac{\theta_{1}(u-\tau v, \tau)}{\eta(\tau)}\right|\left(\prod_{0<q<\lambda} \frac{4 \pi^{2}}{\operatorname{Im}(\tau)^{2}}|(m+u)-\tau(n+v)|^{2}\right)^{-1}
$$

where $q=\frac{4 \pi^{2}}{\operatorname{Im}(\tau)^{2}}|(m+u)-\tau(n+v)|^{2}$, determining the Quillen metric on $\mathscr{L}$ in this case over the open subsets $U_{\lambda}$ of the Jacobian torus.

\section{References}

[1] M. F. Atiyah and I. M. Singer, Dirac operators coupled to vector potentials. Proc. Nat. Acad. Sci. U.S.A. 81 (1984), 2597-2600. Zbl 0547.58033 MR 742394

[2] A. Connes, Noncommutative geometry. Academic Press, San Diego, CA, 1994. Zbl 0818.46076 MR 1303779 
[3] A. Connes and A. H. Chamseddine, Inner fluctuations of the spectral action. J. Geom. Phys. 57 (2006), 1-21. Zbl 1105.58004 MR 2265456

[4] A. Connes and H. Moscovici, The local index formula in noncommutative geometry. Geom. Funct. Anal. 5 (1995), 174-243. Zbl 0960.46048 MR 1334867

[5] A. Connes and M. A. Rieffel, Yang-Mills for non-commutative two-tori. In Operator algebras and mathematical physics (Iowa City, Iowa, 1985), Contemp. Math. 62, Amer. Math. Soc., Providence, RI 1987, 237-266. Zbl 0633.46069 MR 0878383

[6] D. S. Freed, On determinant line bundles. In Mathematical aspects of string theory (San Diego, Calif., 1986), Adv. Ser. Math. Phys. 1, World Sci. Publishing, Singapore 1987, 189-238. Zbl 0751.58036 MR 0915823

[7] J. Fröhlich, O. Grandjean, and A. Recknagel, Supersymmetric quantum theory and non-commutative geometry. Comm. Math. Phys. 203 (1999), 119-184. Zbl 0947.58010 MR 1695097

[8] J. M. Gracia-Bondía, J. C. Várilly, and H. Figueroa, Elements of noncommutative geometry. Birkhäuser Adv. Texts, Birkhäuser, Boston 2001. Zbl 0958.46039 MR 1789831

[9] N. Higson, The residue index theorem of Connes and Moscovici. In Surveys in noncommutative geometry, Clay Math. Proc. 6, Amer. Math. Soc., Providence, RI, 2006, 71-126. Zbl 1120.58017 MR 2277669

[10] H. Moscovici, Eigenvalue inequalities and Poincaré duality in noncommutative geometry. Comm. Math. Phys. 184 (1997), 619-628. Zbl 0874.46044 MR 1462513

[11] D. Perrot, BRS cohomology and the Chern character in noncommutative geometry. Lett. Math. Phys. 50 (1999), 135-144. Zbl 1079.58506 MR 1761171

[12] D. Quillen, Determinants of Cauchy-Riemann operators over a Riemann surface. Funktsional. Anal. i Prilozhen. 19 (1985), no. 1, 37-41; English transl. Functional Anal. Appl. 19 (1985), 31-34. Zbl 0603.32016 MR 783704

[13] D. B. Ray and I. M. Singer, Analytic torsion for complex manifolds. Ann. of Math. (2) 98 (1973), 154-177. Zbl 0267.32014 MR 0383463

Received December 15, 2008

P. S. Chakraborty, Department of Mathematics, University of Adelaide, Adelaide 5005, Australia, (On leave from The Institute of Mathematical Sciences, Chennai, India)

E-mail: partha.chakraborty@adelaide.edu.au,parthac@imsc.res.in

V. Mathai, Department of Mathematics, University of Adelaide, Adelaide 5005, Australia E-mail: mathai.varghese@adelaide.edu.au 